\title{
Bose-Einstein Condensation of Atomic Hydrogen
}

\author{
Dale G. Fried, Thomas C. Killian, Lorenz Willmann, David Landhuis, Stephen C. Moss, Daniel Kleppner, and \\ Thomas J. Greytak \\ Department of Physics and Center for Materials Science and Engineering, Massachusetts Institute of Technology, \\ Cambridge, Massachusetts 02139
}

(Received 11 September 1998)

\begin{abstract}
We report observation of Bose-Einstein condensation (BEC) of a trapped, dilute gas of atomic hydrogen. The condensate and normal gas are studied by two-photon spectroscopy of the $1 S-2 S$ transition. Interactions among the atoms produce a shift of the resonance frequency proportional to density. The condensate is clearly distinguished by its large frequency shift. The peak condensate density is $4.8 \pm 1.1 \times 10^{15} \mathrm{~cm}^{-3}$, corresponding to a condensate population of $10^{9}$ atoms. The BEC transition occurs at about $T=50 \mu \mathrm{K}$ and $n=1.8 \times 10^{14} \mathrm{~cm}^{-3}$. [S0031-9007(98)07617-0]

PACS numbers: 03.75.Fi, 05.30.Jp, 32.80.Pj, 67.65.+z
\end{abstract}

The search for Bose-Einstein condensation (BEC) in dilute atomic gases began in earnest in 1978 [1], precipitated by the suggestion of Stwalley and Nosanow [2] that spin-polarized atomic hydrogen should be an ideal candidate for the study of such extreme quantum behavior. Dilute gases of spin-polarized $\mathrm{H}$ were first stabilized by Silvera and Walraven [3]. Subsequent attempts to achieve BEC were thwarted by recombination on the walls of the confinement cell [4]. In order to create the colder samples necessary for further progress toward BEC, our group suggested [5] and demonstrated [6] wall-free confinement and evaporative cooling. Evaporative cooling made it possible to cool alkali metal atoms below the laser cooling limit and to achieve BEC in dilute gases of Rb [7], $\mathrm{Na}$ [8], and $\mathrm{Li}$ [9]. In this paper we report the achievement of $\mathrm{BEC}$ in $\mathrm{H}$ and a study of the gas by high resolution spectroscopy.

Hydrogen condensates share many characteristics with condensates of alkali metal atoms, but there are several notable differences. Owing to hydrogen's simplicity, properties such as interatomic potentials and spin relaxation rates are well understood theoretically. Compared to those of other atoms, the $s$-wave scattering length, $a$, and three-body loss rate are anomalously low. As a result the condensate density is high, even for small condensate fractions, and the elastic collision rate is low, which retards evaporative cooling. Because of hydrogen's small mass, the BEC transition occurs at higher temperatures than previously observed. The cryogenic trap loading technique used for $\mathrm{H}$ allows orders of magnitude more atoms to be trapped, providing condensates that are much larger than yet achieved in other systems. Finally, high resolution two-photon spectroscopy provides a new tool for studying condensates.

The starting point for the observations reported here is described in the accompanying Letter [10]: a gas of atomic hydrogen, confined in a cylindrically symmetric magnetic trap, and cooled to $120 \mu \mathrm{K}$ by evaporation over a magnetic field saddlepoint at one end of the trap [11]. Below about $120 \mu \mathrm{K}$ this method of forcing evaporation proved to be inefficient. As explained by Surkov et al. [12], when the temperature is reduced and the atoms settle into the harmonic region of the trap, the axial and radial motional degrees of freedom become uncoupled. For efficient evaporation, the escape time for energetic atoms must be short compared to the collision time. However, the escape time for atoms with high radial energy becomes very long when the motion uncouples, and thus escape is blocked for this large class of energetic atoms. By itself this would only slow evaporation, but in the presence of losses due to dipolar relaxation $[5,6]$ phase space compression ceases.

To overcome this problem, we turned to a different ejection technique, based on spin resonance [13], first demonstrated for the alkali metal atoms [14]. An rf field causes transitions to an untrapped magnetic sublevel wherever the trapping magnetic field satisfies the resonance condition. Atoms with high energy in any degree of freedom are thus able to escape, ensuring high evaporation efficiency. As the rf frequency is decreased, the energy threshold for ejection from the trap is lowered. To implement rf evaporation in the cryogenic environment, we developed a nonmetallic trapping cell which allows us to apply rf magnetic fields with strength up to $2 \times 10^{-7} \mathrm{~T}$ and frequency up to $46 \mathrm{MHz}$. Typically, we switch to rf evaporation at a trap depth of $1.1 \mathrm{mK}$, corresponding to a sample temperature of $120 \mu \mathrm{K}$.

We measure the temperature and density distribution of the gas by two-photon spectroscopy of the $1 S-2 S$ transition [15]. Two-photon excitation in a standing wave leads to two types of absorption: Doppler-sensitive, due to absorption of copropagating photons, and Dopplerfree, due to absorption of counterpropagating photons. In the first case the spectrum exhibits a recoil shift of $\hbar k^{2} / 2 \pi m=6.7 \mathrm{MHz}$ and Doppler broadening that can be used to measure the absolute temperature. In the second case, Doppler-free excitation results in a narrow, intense line. These features are shown in the composite spectrum (Fig. 1). 


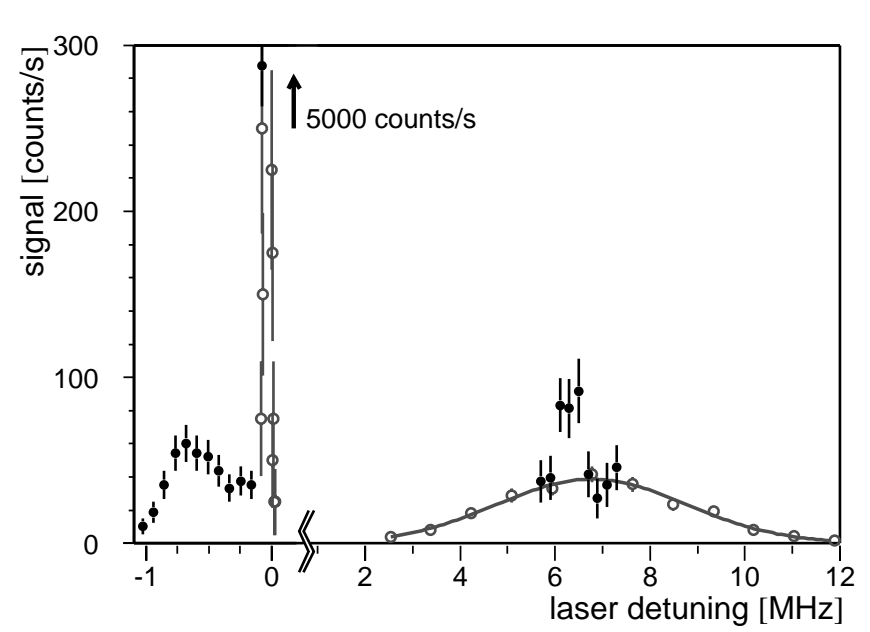

FIG. 1. Composite $1 S-2 S$ two-photon spectrum of trapped hydrogen. (Note change in frequency scale near the origin.) O: Spectrum of sample without a condensate; 0 : spectrum emphasizing features due to a condensate. The intense, narrow peak arises from absorption of counterpropagating photons by the normal gas, and exhibits no first-order Doppler broadening. The wide, low feature on the right is from absorption of copropagating photons. The solid line is the recoil-shifted, Doppler-broadened, Gaussian line shape of the normal gas corresponding to $T=40 \mu \mathrm{K}$. The high density in the condensate shifts a portion of the Doppler-free line to the red. The condensate's narrow momentum distribution gives rise to a similar feature near the center of the Doppler-sensitive line. Zero detuning is taken for unperturbed atoms excited Doppler-free. All frequencies are referenced to the $243 \mathrm{~nm}$ excitation radiation.

When the sample is cooled below $50 \mu \mathrm{K}$, additional features appear in the spectrum, indicating the onset of BEC. The Doppler-free spectrum exhibits a new feature with a large redshift. As described in the accompanying Letter [10], the $1 S-2 S$ transition frequency is redshifted by an amount proportional to the density. Thus, this new feature indicates the formation of a region of high density, as expected for a condensate. Another new feature also appears in the Doppler-sensitive spectrum, which probes the sample's momentum distribution; the narrow momentum distribution of the condensate appears as an additional feature in the middle of the wide, recoilshifted Doppler profile of the thermal cloud [16]. This feature, whose intrinsic width should be given by the position-momentum uncertainty relation, is broadened in these experiments by the high condensate density. As expected, it appears qualitatively similar to the Dopplerfree spectrum of the condensate.

The onset of BEC is further confirmed by the trajectory of the normal gas as it is cooled through phase space (see Fig. 2). The maximum density of the normal gas, $n_{0}$, is determined by measuring the shift of the Doppler-free spectrum of the normal gas [10]. As the trap depth, $\boldsymbol{\epsilon}_{t}$, is reduced from $1.1 \mathrm{mK}$ to $300 \mu \mathrm{K}$, the density increases because the cooled atoms settle into the lowest energy regions of the trap. At a depth of $300 \mu \mathrm{K}$ there is a change in the trajectory; it begins to fall with temperature, indicating the onset of BEC. For a sample in equilibrium below the transition temperature, the maximum density of the normal fraction is pinned at the critical density $n_{c}(T)=2.612\left(2 \pi m k_{B} T\right)^{3 / 2} / h^{3}$; excess population is in the Bose condensate. Although it is not clear that our samples are in thermal equilibrium, this portion of the trajectory approximates the BEC phase transition line.

The solid line in Fig. 2 indicates the critical density, $n_{c}(T)$, assuming that $\eta \equiv \epsilon_{t} / k_{B} T=6$. The parameter $\eta$ is set by a competition between cooling due to evaporation and heating due to dipolar spin relaxation. Dipolar relaxation preferentially removes low energy atoms from regions of highest density at the bottom of the trap. The condensate has a high density, and thus high loss rate. The value of $\eta$ is expected to change from about 9 at higher temperatures in the absence of a condensate to about 5 in the presence of a condensate at the lowest trap depths. The value $\eta=6$ was measured spectroscopically at a trap depth $\epsilon_{t}=280 \mu \mathrm{K}$ [17].

Figure 3 is an enlarged view of the Doppler-free spectrum of a Bose-condensed sample. The high narrow feature is due to excitation of the normal gas. The condensate portion of the spectrum extends to at least $-0.9 \mathrm{MHz}$. To extract the condensate density we assume that the linear relation between frequency shift and density for a normal gas found in Ref. [10] remains valid at high densities. For excitation out of a Bose-Einstein condensate, one might expect that the disappearance of exchange effects would reduce the frequency shift to one half of that for a normal gas at the same density. However, this reduction may not occur if the system condenses

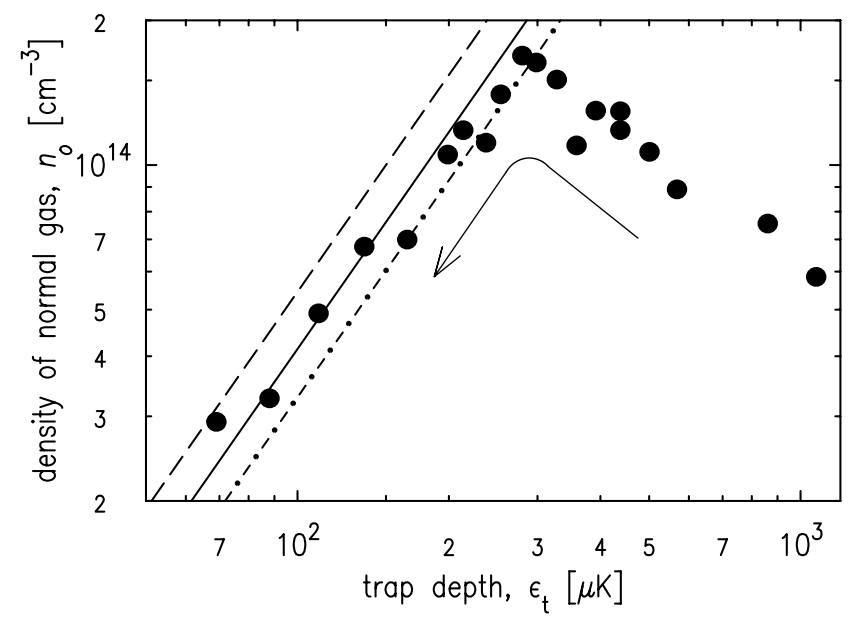

FIG. 2. Density of noncondensed fraction of the gas as the trap depth is reduced along the cooling path. The density is measured by the optical resonance shift [10], and the trap depth is set by the rf frequency. The lines (dashed, solid, dot-dashed) indicate the BEC phase transition line, assuming a sample temperature of $\left(\frac{1}{5}\right.$ th, $\frac{1}{6}$ th, $\frac{1}{7}$ th) the trap depth. The scatter of the data reflects the reproducibility of the laser probe technique and is dominated by the alignment of the laser beam to the sample. 


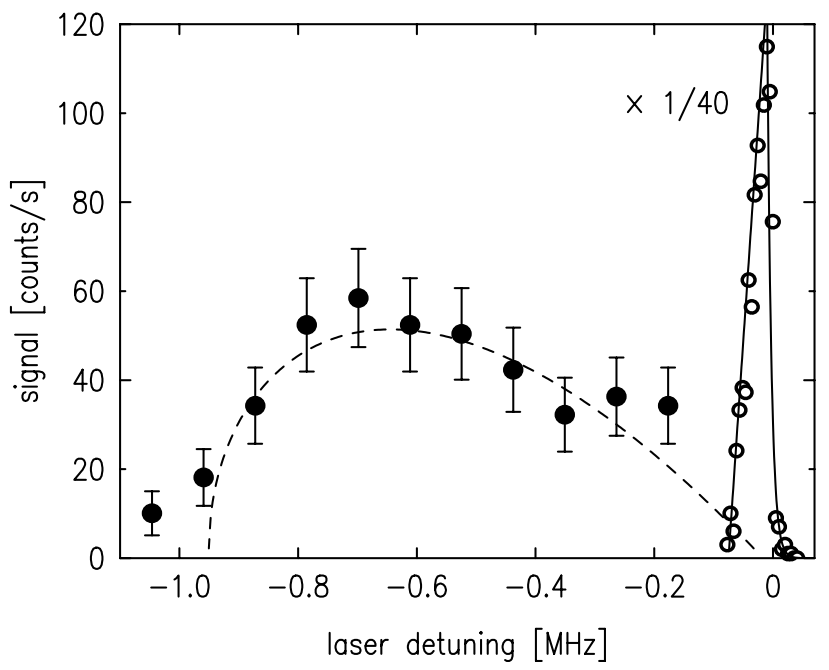

FIG. 3. Doppler-free spectrum of the condensate (broad feature) and the normal gas (narrow feature). The dashed line is proportional to the number of condensate atoms at a density proportional to the detuning, for an equilibrium density distribution with peak density $n_{p}=4.8 \times 10^{15} \mathrm{~cm}^{-3}$ in a parabolic trap.

into a group of low-lying states [18], in which case the frequency shift is the same as for a normal gas [19]. Using this conservative assumption, for two-photon excitation, $\Delta \nu_{1 S-2 S}=2 \Delta \nu_{243 \mathrm{~nm}}=\chi n$, where $\chi=-3.8 \pm$ $0.8 \times 10^{-16} \mathrm{MHz} \mathrm{cm}^{3}$. We derive a peak condensate density of $n_{p}=4.8 \pm 1.1 \times 10^{15} \mathrm{~cm}^{-3}$. The spectrum depends on the distribution of densities in the condensate, and thus the magnitude squared of the condensate wave function.

The peak condensate density extracted from Fig. 3 is the largest in this series of experiments, but several other spectra gave comparable results. From this peak density we compute the mean field energy, the total number of condensate atoms, and the condensate fraction. We model our trap by the Ioffe-Pritchard potential $V(\rho, z)=\sqrt{(\alpha \rho)^{2}+\left(\beta z^{2}+\theta\right)^{2}}-\theta$. The parameters $\alpha$ and $\beta$ are calculated from the magnetic coil geometry. Because of the sensitivity of the axial bias, $\theta$, to stray fields, we measure it using an rf ejection technique; for these experiments $\theta / k_{B}=35 \pm 2 \mu \mathrm{K}$. For small displacements the radial oscillation frequency is $\omega_{\rho}=\alpha / \sqrt{m \theta}=2 \pi \times 3.90 \pm 0.11 \mathrm{kHz}$, and the axial frequency is $\omega_{z}=\sqrt{2 \beta / m}=2 \pi \times 10.2 \mathrm{~Hz}$. The peak mean field energy $n_{p} \tilde{U} / k_{B}=n_{p} h^{2} a / \pi m k_{B}=1.9 \mu \mathrm{K}$ is much greater than the harmonic oscillator level spacing, $\hbar \omega_{r} / k_{B}=190 \mathrm{nK}$.

As the simplest model of the interacting gas, we assume the Thomas-Fermi density profile for the condensate, $n(\rho, z)=n_{p}-V(\rho, z) / \tilde{U}$. The number of atoms in the condensate is found by integrating the density over the volume of the condensate. The result is $N_{c}=$ $16 \pi \sqrt{2} \tilde{U}^{3 / 2} n_{p}^{5 / 2} / 15 \omega_{\rho}^{2} \omega_{z} m^{3 / 2}=1.1 \pm 0.6 \times 10^{9}$. This condensate is $15 \mu \mathrm{m}$ in diameter and $5 \mathrm{~mm}$ in length.
Compared to alkali metal atoms, the maximum achievable equilibrium condensate fraction for hydrogen is expected to be small [20]. Hydrogen's small $s$-wave scattering length dramatically reduces the elastic collision rate in the thermal cloud, and thus the evaporative cooling rate. The high density in a condensate can drastically increase the heating due to dipolar relaxation; the resulting heatingcooling balance limits the condensate fraction.

The condensate fraction, $f \equiv N_{c} /\left(N_{n}+N_{c}\right)$, is obtained by comparing the integrated area of the spectra of the condensate and normal gas after accounting for the partial $(\sim 10 \%)$ illumination of the normal gas by the laser beam. A condensate fraction $f=5_{-2}^{+4} \%$ is obtained, with the dominant uncertainty coming from the relative size of the laser beam and thermal cloud. Alternatively, the condensate fraction may be estimated by comparing the condensate population, $N_{c}$, to the calculated normal gas population, $N_{n}$, which is simply the Bose occupation function weighted by the density of states, integrated over the trapped states. The trap parameters $\alpha$ and $\beta$ cancel in the ratio $N_{c} / N_{n}$, and the remaining parameters, $T, n_{p}$, and $\theta$, are measured. We use the Doppler-broadened spectrum to measure the temperature at $45 \pm 5 \mu \mathrm{K}$. Since this thermal energy scale is much larger than the peak mean field energy, perturbations to the density distribution of the normal gas due to the condensate may be neglected. The resulting fraction is $f=6_{-3}^{+6} \%$. Both methods for determining $f$ assume thermal equilibrium, which may not be the case because the mean collision time is comparable to the axial oscillation period.

Note that if we had assumed that the frequency shift in the condensate is only half as large as for a normal gas at the same density, as one would expect for a condensate in a single quantum state, the density extracted from the spectrum would be twice as large. This would imply that $N_{c}=6 \times 10^{9}$ and yield an unreasonably high condensate fraction of $25 \%$.

We have followed the time evolution of individual condensates by recording series of spectra in rapid succession. As expected, when the condensate decays the spectrum narrows, indicating a reduction in density, and becomes less intense, indicating a reduction in condensate population. The lifetime is observed to be about $5 \mathrm{~s}$. The condensate decays rapidly by dipolar relaxation, but is simultaneously replenished by the normal gas. The laser probe artificially shortens the lifetime by evaporating $\mathrm{He}$ from optical surfaces; the streaming He knocks atoms out of the trap.

We find that the presence of the condensate significantly distorts the Doppler-free spectrum of the normal gas, even though the condensate volume is only about $10^{-3}$ of the volume of the thermal cloud. Figure 4 shows spectra taken above and below the onset of BEC. The spectra for samples without condensates show the shift and line shape due to the inhomogeneous density. The lines are roughly symmetric, and are consistent with 


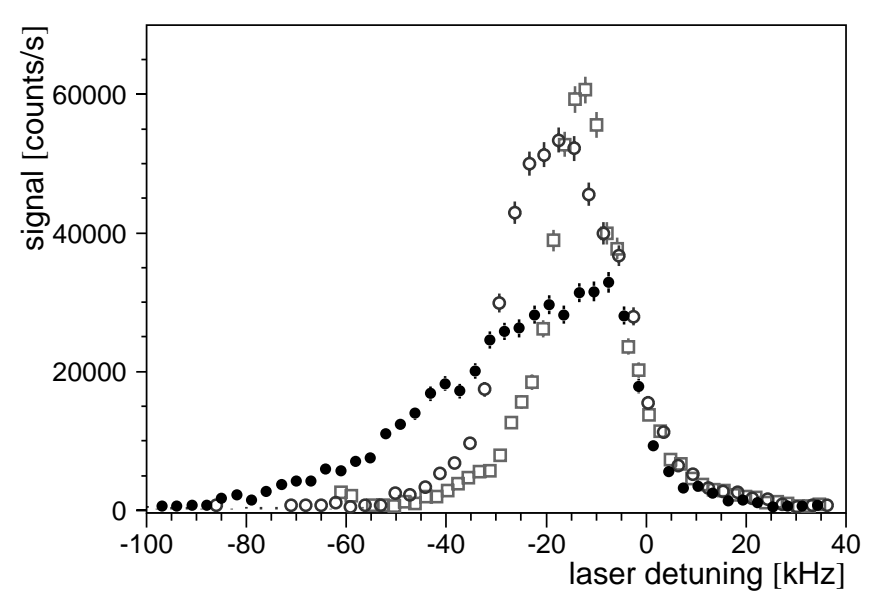

FIG. 4. Doppler-free spectrum of normal fraction above and below the onset of BEC. The symmetric spectrum (above $T_{c}$, open symbols) suddenly becomes asymmetric (solid symbols) when the condensate forms. Temperatures for the three spectra are about $120 \mu \mathrm{K}$ (open squares), $53 \mu \mathrm{K}$ (open circles), and $44 \mu \mathrm{K}$ (solid circles).

calculations assuming thermal equilibrium. Below the transition, however, a pronounced asymmetry appears, showing spectral weight at frequency shifts much larger than expected for the maximum density in the normal gas, $n_{c}(T)$. The origin of this effect is not yet understood.

The signal-to-noise ratio in these experiments is limited by the light collection solid angle, about $2 \times 10^{-2}$ sr. Improvements should allow the spectroscopic observation technique to provide information which is complementary to that obtained by the spatial imaging techniques used in other experiments; the Doppler-sensitive spectrum constitutes an in situ direct measure of the momentum distribution.

Many people played important roles in the MIT work on spin-polarized hydrogen over the years. We especially wish to acknowledge those who contributed directly to the trapping and spectroscopy experiments: Claudio L. Cesar, John M. Doyle, Harald F. Hess, Greg P. Kochanski, Naoto Masuhara, Adam D. Polcyn, Jon C. Sandberg, and Albert I. Yu. We thank Wolfgang Ketterle and Leonid Levitov for helpful discussions. This research is supported by the National Science Foundation and the Office of Naval Research. The Air Force Office of Scientific Research contributed in the early phases. L.W. acknowledges support from Deutsche Forschungsgemeinschaft. D. L. and S.C.M. are grateful for support from the National Defense Science and Engineering Graduate Fellowship Program.

Note added.-Observation of quantum degeneracy in a two-dimensional hydrogen system has been reported recently [21].
[1] Session on trapped atomic hydrogen, Bull. Am. Phys. Soc. 23, 85 (1978).

[2] W.C. Stwalley and L.H. Nosanow, Phys. Rev. Lett. 36, 910 (1976).

[3] I.F. Silvera and J. T. M. Walraven, Phys. Rev. Lett. 44, 164 (1980).

[4] D. A. Bell, H. F. Hess, G. P. Kochanski, S. Buchman, L. Pollack, Y. M. Xiao, D. Kleppner, and T. J. Greytak, Phys. Rev. B 34, 7670 (1986), and references therein.

[5] H. Hess, Phys. Rev. B 34, 3476 (1986).

[6] N. Masuhara, J. M. Doyle, J.C. Sandberg, D. Kleppner, T. J. Greytak, H.F. Hess, and G. P. Kochanski, Phys. Rev. Lett. 61, 935 (1988).

[7] M.H. Anderson, J.R. Ensher, M.R. Matthews, C.E. Wieman, and E. A. Cornell, Science 269, 198 (1995).

[8] K. B. Davis, M.-O. Mewes, M.R. Andrews, N. J. van Druten, D. S. Durfee, D. M. Kurn, and W. Ketterle, Phys. Rev. Lett. 75, 3969 (1995).

[9] C. C. Bradley, C. A. Sackett, J. J. Tollett, and R. G. Hulet, Phys. Rev. Lett. 75, 1687 (1995); C.C. Bradley, C. A. Sackett, and R.G. Hulet, Phys. Rev. Lett. 78, 985 (1997).

[10] T. C. Killian, D. G. Fried, L. Willmann, D. Landhuis, S. C. Moss, T. J. Greytak, and D. Kleppner, preceding Letter, Phys. Rev. Lett. 81, 3807 (1998).

[11] T. J. Greytak, in Bose-Einstein Condensation, edited by A. Griffin, W.W. Snoke, and S. Stringari (Cambridge University Press, Cambridge, England, 1995), p. 131.

[12] E. L. Surkov, J. T.M. Walraven, and G. V. Shlyapnikov, Phys. Rev. A 49, 4778 (1994); Phys. Rev. A 53, 3403 (1996).

[13] D.E. Pritchard, K. Helmerson, and A.G. Martin, in Atomic Physics 11, edited by S. Haroche, J. C. Gay, and G. Grynberg (World Scientific, Singapore, 1989), p. 179.

[14] W. Petrich, M.H. Anderson, J.R. Ensher, and E. A. Cornell, Phys. Rev. Lett. 74, 3352 (1995); K. B. Davis, M.O. Mewes, M. A. Joffe, M. R. Andrews, and W. Ketterle, Phys. Rev. Lett. 74, 5202 (1995).

[15] C. L. Cesar, D. G. Fried, T. C. Killian, A. D. Polcyn, J.C. Sandberg, I. A. Yu, T. J. Greytak, D. Kleppner, and J. M. Doyle, Phys. Rev. Lett. 77, 255 (1996).

[16] Jon C. Sandberg, Ph.D. thesis, Massachusetts Institute of Technology, 1993.

[17] At lower densities the low signal rate precludes the use of the Doppler-sensitive spectrum for measuring temperature.

[18] Yu. M. Kagan, B. V. Svistunov, and G. V. Shlyapnikov, Sov. Phys. JETP 75, 387 (1992); C. W. Gardiner, M. D. Lee, R.J. Ballagh, M. J. Davis, and P. Zoller, http:// xxx.lanl.gov/abs/cond-mat/9806295.

[19] Leonid Levitov (private communication).

[20] T. W. Hijmans, Y. Kagan, G. V. Shlyapnikov, and J. T. M. Walraven, Phys. Rev. B 48, 12886 (1993).

[21] A.I. Safonov, S. A. Vasilyev, I. S. Yasnikov, I. I. Lukashevich, and S. Jaakkola (private communication); http://publish.aps.org/eprint/gateway/epsearch/ aps1998sep10_001. 